\title{
SYSTEMATIC REVIEW OF EDUCATIONAL INTERVENTIONS TO IMPROVE THE UPTAKE OF POST-PARTUM INTRAUTERINE CONTRACEPTIVE DEVICE (PPIUCD)
}

\author{
Saima', Julie Cullen², Syed Mustafa Ali ${ }^{3}$ \\ ${ }^{1}$ Post-graduate student, University of Southampton, UK \\ ${ }^{2}$ Principal Teaching Fellow: Faculty of Health Sciences, University of Southampton, UK, email: j.cullen@soton.ac.uk \\ ${ }^{3}$ Syed Mustafa_Ali, mHealth Coordinator, Mercy Corps, email: Mustafa_30_84@yahoo.com \\ Correspondence: Saima, email: dr.saimalakhani@gmail.com, Cell: 00923432128378
}

\begin{abstract}
Background: Short inter-pregnancy intervals result in poor peri-natal outcomes and an increased uptake of Post Partum Intra-Uterine Contraceptive Device (PPIUCD) can help preventing adverse peri-natal outcomes.

Search Strategy: Throughout June 2014, AMED , CINAHL Plus, Cochrane Library, EMBASE, MEDLINE (EBSCO), and PsycINFO, were searched using Medical Subject Heading (MeSH) and truncation. Peer reviewed and published articles in past 10 years (2004 to 2014) measuring the improvement in the uptake of PPIUCD through educational interventions have been included in the review. The results of the relevant studies are retrieved by the author and reviewed by the project supervisor to check the relevance of selected evidence. The analysis is carried out by appraising data for similar interventions.

Results: Eight studies, accumulatively representing 88071 participants, met the inclusion criteria, including three RCTs, two systematic reviews, one qualitative study, one cross-sectional study and a cohort study. Five studies on educational interventions of postpartum females have showed an increase in uptake of IUCD. Two studies on providers' training have also showed improvement in PPIUCD uptake. However, one study educating postpartum females did not show improved uptake.

Key Words: Behavior change communication, educational intervention, ppiucd uptake, strategies to improve ppiucd uptake, training of counselors, patient education, intrauterine device, ppiucd.
\end{abstract}

\section{Introduction}

In Pakistan, the population is growing rapidly which is not matched with the contraceptive prevalence rate (1). Therefore, there is an indispensable need to promote contraceptive use. However, Behavior Change Communication (BCC) is identified as one of the major ingredients in the recipe of successful Family Planning (FP) project design 2,3.

National Institute of Population Studies Pakistan reports that 34 percent of the deliveries in Pakistan occur within 24 months of last delivery 1. Conversely, World Health Organization guidelines for safe motherhood state that a couple must have a gap of at least 2 years between last delivery and next conception 4 . There is a direct relationship between low birth intervals and poor maternal and child health outcomes5, which include mineral deficiency (for example, Iron deficiency anemia) and decreased bone mineral density. Additionally, it may also affect the maternal psychological condition by increasing the stress of taking care of newborn 4.

Postpartum period is the ideal time to commence contraception 6 and its uptake provides an exclusive chance to cater high unmet needs of FP 7. Abiodun et al. identify the antenatal visits as an opportunity to counsel women for using postpartum FP method 8 . However, in Pakistan, 73 percent of women attend antenatal clinics of skilled providers, and 28 percent women attend at least four antenatal visits 1 . Therefore, integrating FP services with safe motherhood services can increase the chances for postpartum contraception uptake after the delivery 9,10 because it allows woman to decide for appropriate birth interval to replenish her body needs 7 . Usually postpartum contraception is delayed until 6 weeks after delivery and women are discharged from hospital with an advice to avoid sexual activity which puts women on risk of an unintended pregnancy 11. Sexual activity resumed earlier and ovulation of nonbreastfeeding women starting from 4th week postpartum put women at higher risk for unintended pregnancy 12. Therefore, to eliminate the chances of occurrence of unintended pregnancy, PPIUCD is the most effective contraceptive method $11,13,14$. 
PPICUD adoption offers several advantages, including decreased chances of unintended pregnancy 11, noninterference with hormonal balance of breastfeeding mothers 15 and convenience for both women and service providers because of less discomfort associated with its use 13. Despite the benefits of PPIUCD its uptake so far is very low, which is explained by the lack of user's knowledge, provider's knowledge and lack of appropriate technical skills to provide postpartum contraceptive counseling and services 13-15. The key objective of the review is to evaluate the effectiveness of educational interventions implemented globally in improving the uptake of PPIUCD and their feasibility of implementation in the context of Pakistan and to inform the development of future projects.

\section{Methodology}

The PICO methodology was adopted to frame a specific answerable question for this systematic review (Figure 1).

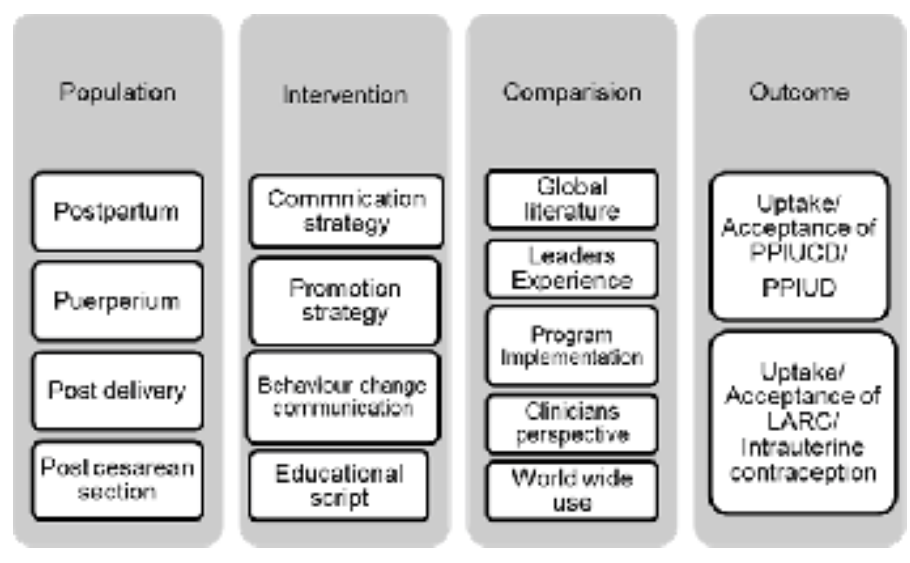

Figure 1: PICO Strategy and Keywords

\section{Literature Search}

AMED (Allied and Complementary Medicine Database), CINAHL Plus (Cumulative Index of Nursing and Allied Health Literature), Cochrane Library, EMBASE, MEDLINE (EBSCO), and PsycINFO computerized bibliography databases were searched for peer reviewed studies published between 1 January 2004 and 3 June 2014 in English language. The literature search was carried out in June 2014 using keywords corresponded to four elements of PICO strategy: Population, Intervention, Comparison and Outcome as shown in Figure 1. Boolean operators "AND" and "OR" were used to connect the keywords to achieve accurate results.

Using Boolean operators, the sample search strings used for Medline are given below:

String 1: (((behaviour change communication) OR educational intervention) OR training of service providers) OR patient education Filters: Publication date from 2004/01/01 to 2014/06/31

Output of String $1=93916$ articles

String 2: Search ((((intrauterine device) OR post partum intrauterine device) OR larc) OR increased uptake of larc) OR postpartum family planning Filters: Publication date from 2004/01/01 to 2014/06/31

Output of String $2=2350$ articles

String 3: Search (( (( (behaviour change communication) OR educational intervention) OR training of service providers) OR patient education) AND ( "2004/01/01"[PDat] : "2014/06/31"[PDat] ))) AND ((()((intrauterine device) OR post partum intrauterine device) OR larc) OR increased uptake of larc) OR postpartum family planning) AND ( "2004/01/01"[PDat] : "2014/06/31"[PDat] )) Filters: Publication date from $2004 / 01 / 01$ to $2014 / 06 / 31$

Output of String $3=$ String 1 AND String $2=180$ articles

\section{Eligibility Criteria}

All randomized controlled trials, systematic reviews, cohort studies, case control studies and qualitative studies were included in the review if they: (1) reported women given birth to a child by either cesarean section or vaginal delivery (2) reported the use of Intra-uterine contraceptive device (IUCD). However, the inclusion criteria are presented in the Figure 2.

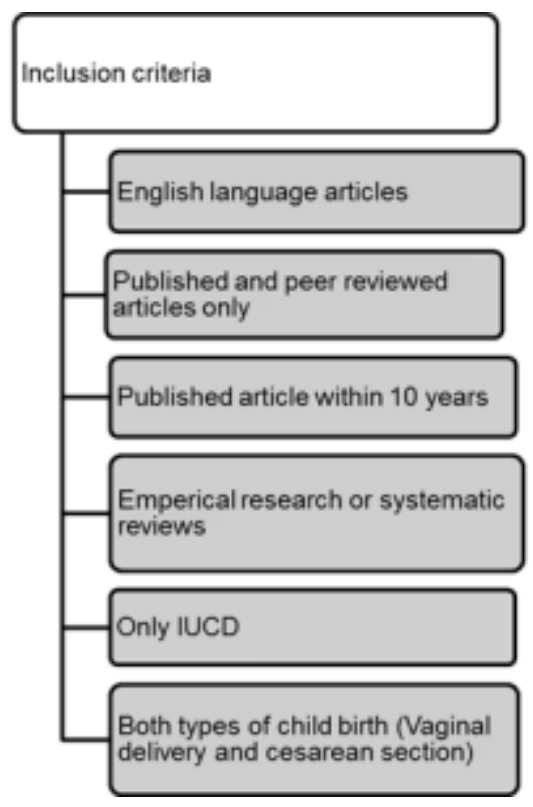

\section{Selection Results}

Initial databases search yielded 328 articles. After screening the title 91 studies were selected. The abstract screening was performed as a next stage which helped refining selected evidence to 38 articles. Full texts were retrieved and critically appraised by using Critical Appraisal Skill Program (CASP) tools to evaluate the suitability of evidence in answering the clinical question. The articles lacking scientific rigor were rejected with the help of CASP tool. This process culminated in the final selection of 8 articles for systematic review. Figure 3 shows the process of selection of relevant evidence. 


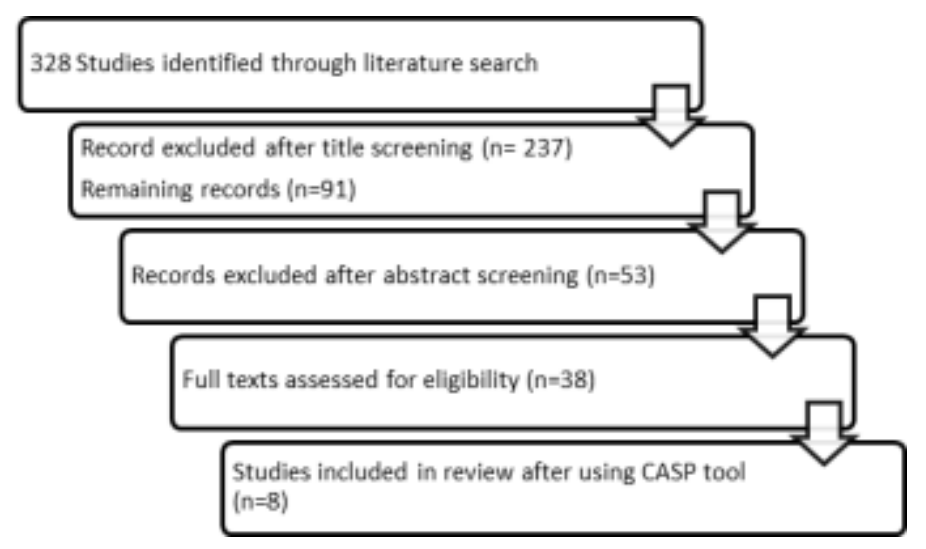

Figure 3: Result of Systematic Search Justifications for Eligibility and Inclusion Criteria All the articles selected as evidence are in English language to avoid misinterpretation. The articles selected are peer reviewed and published in journal; no unpublished article has been included. Empirical researches, including both qualitative and quantitative studies and systematic reviews are included because they are considered credible in hierarchy of evidence 16. We applied pre-defined systematic review protocol to search strategy and search results were excluded of any selection bias. We included qualitative studies in eligibility criteria as they are exploratory in nature and can help understanding behavior, preferences and educational needs of the participants.

\section{Results}

A total of eight studies, accumulatively representing 88071 participants (1256 counselors and 86815 service consumers) met the inclusion criteria. The counselors had different professional levels including doctors, community workers and lady health workers. The selected studies were peer reviewed, published and have discussed $\mathrm{BCC}$ strategies in postpartum women using IUCD18-25. The table 1 below shows the characteristics of each study included in the review

Table 1. Characteristics of the included studies

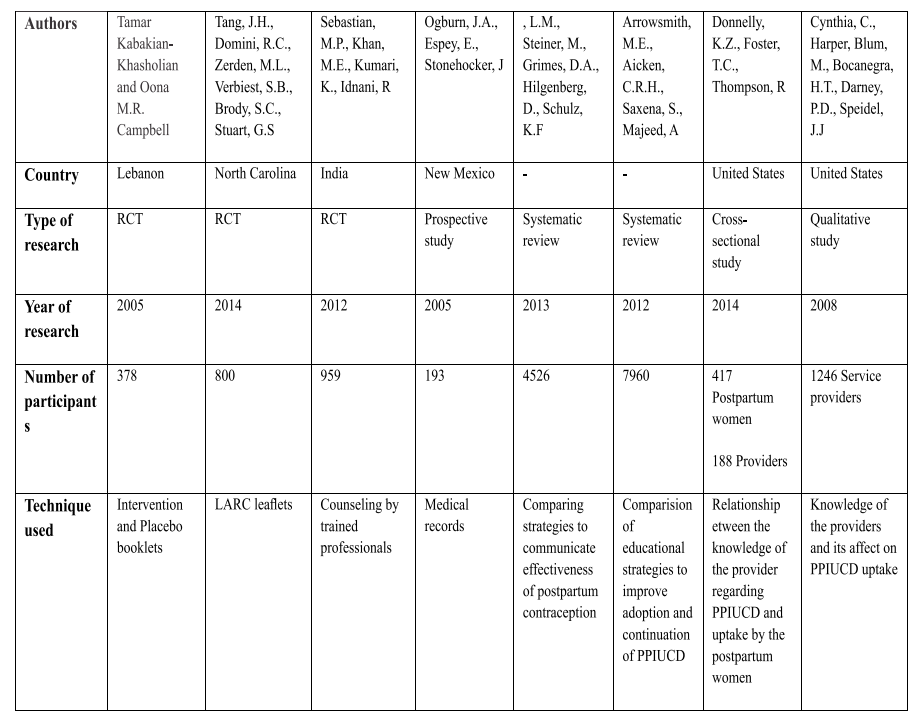

Overall, the studies included in the review fell into two main types of BCC based educational interventions: (1) education of postpartum women through community based intervention and (2) education of counselors, who provide counseling session to women. Due to differences or heterogeneity in the interventions and participants, the results cannot be combined in the review. Sub-group analysis was performed for each type of intervention for the purpose of result formulation.

\section{Educational Intervention for Postpartum Women}

In total, seven different studies have discussed educational interventions for postpartum women and have measured the effects of the intervention. The research design and details of intervention are mentioned below for respective studies;

Kabakian-Khasholian and Campbell 18 conducted a single blinded randomized control trial aimed at changing knowledge, attitude and behavior of women regarding postpartum contraception by a specially prepared booklet in Arabic. The study took place at four hospitals in Lebanon recruiting 378 postpartum women. Eight trained female interviewers collected the baseline data. On discharge, interviewers handed each woman a sealed and numbered envelope containing the intervention booklet or the placebo leaflets. Interviewers were blinded about the allocation group of women. Interviewers conducted follow up assessment at women's residence 6-20 weeks after delivery.

Tang et al. 20 performed a randomized controlled trial engaging 800 postpartum women in North Carolina. Women were selected from postpartum unit and were randomized to intervention or the control group. The intervention group received Long Acting Reversible Contraception (LARC) scripts prior to hospital discharge. Follow up phone calls were made to assess LARC use. Pearson Chi Square test was performed to compare LARC use between both arms.

Sebastian et al. 21 studied the impact of behavior change communication intervention integrated into ongoing government program. The intervention was aimed at increasing the knowledge and use of postpartum contraception through counseling by community workers. The intervention took place in 2007 , involving 959 women residing in Uttar Pradesh, India. The results were analyzed by measuring difference in knowledge and contraception use between the baseline and four and nine month postpartum follow- up among intervention and comparison groups.

Ogburn et al. 22 studied the barriers to PPIUCD uptake in postpartum women who delivered at University of New Mexico. The study involved 193 women who desired to use IUCD as a method of contraception on their hospital discharge. Medical records were used to identify the timing of IUCD placement or reason for refusal to uptake the services.

Lopez et al. 23 reviewed seven randomized controlled trials comparing strategies for communicating the effectiveness of postpartum contraception to the 
consumers. The review study included 4526 postpartum women. Outcome was measured in terms of improvement in knowledge of contraceptive effectiveness and attitude towards particular family planning method (i.e. PPIUCD).

Arrowsmith et al. 24 carried out a systematic review to determine the effectiveness of various educational strategies to improve the adoption and continuation in use of IUCD. The review included nine studies representing 7960 women and the results were generated by categorizing the interventions into sub groups.

Donnelly et al. 25 performed a cross-sectional study by administering online convenience survey to identify the priorities of women and contraceptive providers for contraceptive decision making and counseling. A total of 417 women and 188 contraceptive service providers participated in the study. Participants were provided with a list of 34 questions regarding the features on PPIUCD and its importance. The effect of the knowledge provided was measured by reviewing the uptake of PPIUCD.

\section{Overall Findings of Educational Interventions for Postpartum Women}

Seven studies, with an aggregated sample size of 15171 individuals, reported the impact of contraceptive counseling on the uptake of PPIUCD 18,20-25. A greater increase in IUCD uptake is seen in the postpartum women who were provided with the information about the PPIUCD. Conversely, no effect of educational material on uptake of PPIUCD has been reported by Tang et al. 20.However, techniques utilized by the studies are different therefore comparison of different methods and meta-analysis cannot be performed. Overall, statistically significant improvement in PPIUCD uptake was observed by educating women regarding postpartum contraception.

\section{Educational Intervention for the Counselors}

Cynthia et al. 19 evaluated the knowledge of physicians, nurse practitioners and physician assistants serving about 100 contraception consumers per year under California Family Planning Program. A total of 816 service providers participated in the study. Multiple logistic regression was used to measure the association between knowledge and clinical practice of respective practicing group i.e., physicians, nurses and assistants. The available evidence regarding the effective strategies to help postpartum female acquire PPIUCD is limited. One trial and two systematic reviews showed that educational material provided to the postpartum female were useful in enhancing the uptake of PPIUCD. Whereas one trial identified no difference in uptake of PPIUCD linked to educational material provided. Moreover, other three included studies showed that the counseling session by the provider plays a major role in uptake of PPIUCD. Strategies for communicating contraceptive information should be tested in clinical settings and assessed for effect on uptake of contraception.

\section{Overall Findings of Educational Interventions for Providers}

Cynthia et al. 19 studied impact of training on PPIUCD uptake by educating physicians, physician assistants and nurse practitioners. $92 \%$ of the physicians thought their patient to be receptive to learn about contraceptive methods; however, very few of the doctors were providing information about PPIUCD. 76\% providers either did not offer the IUCD or infrequently provide counseling to the patient due to lack of knowledge 19. The study concluded that after the training of providers, uptake of PPIUCD improved exponentially amongst the postpartum women. The $95 \%$ Confidence Interval is 0.045 which also suggests that the results of the study are significant and correct sample size is used to represent the population. Sebastian et al. 21 measured the effect of educational campaign carried out by trained community workers using leaflets, posters and wall paintings and found campaign to be effective in increasing uptake of PPIUCD. Overall, the improvement was observed in PPIUCD uptake by training the counselors.

\section{Discussion}

The review has identified educational interventions at both levels of users (postpartum women) and providers (counselors) and provided evidence of an increase in the uptake of PPIUCD. The education of women during the pre natal, natal and post natal counseling sessions led to an increase in the uptake of IUCD. However, providing 6 weeks postpartum appointment, before hospital discharge, has also found to be effective in PPIUCD uptake 18. Conversely, in one study, education of postpartum women showed no improvement in PPIUCD adoption after intervention 20.

Intensive contraceptive training of the counselors also showed statistically significant increase in IUCD uptake in postpartum women. The studies included in the review focused on the training and education of the counselors, especially the physicians, for the purpose of provision of postpartum contraception included counseling services $19,21$.

Conclusively, the review suggested the improvement in PPIUCD uptake, both in terms of understanding the mechanism of action of PPIUCD and using it. However, a greater impact of was possibly due to large sample size in studies conducted by Lopez et al. 23 and Arrowsmith et al. 24. Due to an overall increase in the population of third world countries, the importance of knowledge about family planning and adoption of family planning methods are very much emphasized 28 . There are various tools for dispersing the contraceptive information, however, education of counselors and consumers have been studied for its practical implication 23,24. In a randomized controlled trial conducted in Nepal, mothers receiving education on contraceptive methods at the time of delivery were found to be more likely to use PPIUCD as compared to those women who were not provided with contraceptive education 29. This finding 
was in congruence with the studies included in the review in which improvement in uptake of PPIUCD was observed in the intervention group 18,19, 21-23. Another study has concluded that women provided with leaflets regarding contraception (oral and emergency contraceptive pills) have better knowledge about contraception 30 .

In all of the developing countries, contraceptive counselors, field workers and lady health workers are considered backbone of the health care system 31 . Raising their motivation and commitment level by skill and career development incentives may help in improving the overall situation 32. Before this realization, it was thought that women do not go to a medical facility to discuss contraceptive choices and therefore not provided with guidance 31 . However, a study reveals that $70 \%$ of the women, who had their last delivery at tertiary care hospital, had no knowledge about contraception and only $14 \%$ of the women were provided by postnatal advice including contraception 33, which highlights the lack of disseminating contraceptive information by service providers who failed to address birth spacing during antenatal, natal and post-natal visits 33. The situation generates the need for re-evaluation of the role of contraceptive service providers for an effective implementation of family planning programs.

Universally, every couple has knowledge about at least one method of contraception, but the knowledge about its mechanism of action, effectiveness and common side effects is inadequate or missing 34 , whereas, the issue is probably due to the lack of comprehensive knowledge source 34. Information booklets or leaflets may act as a good way to disperse the information but they can never play a role of a counselor as a booklet is unable to discuss women's concerns 31 . These concerns range from simple weight gain to the fear of future infertility after contraceptive use 23. According to a study conducted in Pakistan, menstrual irregularities, increased vaginal discharge and husband's disapproval are the main reasons for discontinuation of IUCD use. Therefore, counseling sessions along with the provision of information leaflets may result in maximum knowledge gain, although it would be time consuming, as reported elsewhere 36 .

\section{Conclusion}

Educational interventions are effective in bringing change in knowledge level of users and provider which results in improved PPIUCD uptake. The shift in family planning method, from short term to long acting reversible method is also noted as an outcome of such educational interventions. Comprehensive contraception information provided by the counseling sessions coupled with dissemination of an educational material has generated greater impact on PPIUCD uptake. Therefore, all the hospitals providing maternity services must provide contraceptive education and counseling services through integrated service model delivered and implemented through well-trained and skilled counselors.

\section{References}

1. National Institute of Population Studies Pakistan (NIPS) and Macro International Incorporated (2013) Pakistan demography and health survey report. Pakistan Demographic and Health Survey 2012-2013. NIPS Health survey. Available from http://www.nips.org.pk/ abstract_files/PDHS\%20Final\%20Report\%20a s\%20of\%20Jan\%2022-2014.pdf [Accessed 13 May 2014]

2. Kotler, P., Roberto, E. L. (1989) Social marketing: Strategies for changing public behavior. Free Press, New York.

3. Calderon, C., Rotaeche, R., Etxebarria, A., Marzo, M., Rico, R., Barandiaran, M. (2006) Gaining insight into the clinical practice guideline development processes: qualitative study in a workshop to implement the grade proposal in Spain. BMC Health Services Research 6(138).

4. World Health Organization (2005) Report of Technical Consultation on Birth Spacing. WHO Maternal, newborn, child and adolescent health. A v a i l a ble from http://www.who.int/maternal_child_adolescent/ documents/birth_spacing05/en/ [accessed 24 February 2014].

5. Levitt, C., Shaw, E., Wong, S., Kaczorowski, J., Springate, R., Sellors, J., et al. (2004) Systematic review of the literature on postpartum care: selected contraception methods, postpartum Papanicolaou test and rubella immunization. Birth 31, 203-212

6. Xu, J.X., Reusche, C., Burdan, A. (1994) Immediate postplacental insertion of the intrauterine device: a review of Chinese and the world's experiences. Advances in Contraception 10, 71-82.

7. Stephenson, R., Hennink, M. (2004) Barriers to Family Planning Service Use among the Urban Poor in Pakistan. Opportunities and Choices working paper.

8. Abiodun, I., Adanikin-Onwudiegwu, U., Olabisi, M.L. (2013) In?uence of multiple antenatal counseling sessions on modern contraceptive uptake in Nigeria. The European Journal of Contraception and Reproductive Health Care 18, 381-387.

9. Berer, M. (2003) Integration of sexual and reproductive health services: A health sector priority. Reproductive Health Matters 11(21), 615.

10. Barber, S.L. (2007) Family planning advice and postpartum contraceptive use among lowincome women in Mexico. International Family Planning Perspectives 33(1), 6-12.

11. Levi, E., Cantillo, E., Ades, V., Banks, E., Murthy, A. (2012) Immediate post placental IUD insertion 
at cesarean delivery: a prospective cohort study. Contraception 86, 102-105.

12. Speroff, L., Mishell, J.D.R. (2008) The postpartum visit: it's time for a change in order to optimally initiate contraception. Contraception 78, 90-98.

13. Kapp, N., Curtis, K.M. (2009) Intrauterine device insertion during postpartum period: A systematic review. Contraception 80:327-336.

14. Eroglu, K., Akkuzu, G., Vural, G.Y., Dilbaz, B., Akvn, A.Y., Ykvn, L.T., et al. (2006) Comparison of efficacy and complications of IUD insertion in immediate postplacental/early postpartum period with interval period: 1 year follow-up. Contraception 74: 376-381.

15. Grimes D, Schulz K, Van Vliet H, Stanwood N. (2001) Immediate postpartum insertion of intrauterine devices. The Cochrane Database of Systematic Review, Issue 2(3036).

16. Burls, A. (2009) What is critical appraisal (2nd edition). Oxford University Press, Oxford.

17. Diaz, Soledad, Croxatto, Horacio, B. (1993) Contraception in lactating women. Current Opinion in Obstetrics and Gynecology 5(6).

18. Kabakian-Khasholian, T., Campbell, O.M.R. (2005) A simple way to increase service use: triggers of women's uptake of postpartum services. British Journal of Obstetrics and Gynecology 112(9), 1315-1321.

19. Cynthia, C., Harper, Blum, M., Bocanegra, H.T., Darney, P.D., Speidel, J.J., et al (2008) Challenges in translating evidence to practice: the provision of intrauterine contraception. American Journal of Obstetricians and Gynecologists 111(6).

20. Tang, J.H., Domini, R.C., Zerden, M.L., Verbiest, S.B., Brody, S.C., Stuart, G.S. (2014) Effect of an educational script on postpartum contraceptive use: a randomized controlled trial. Contraception 90 (2), 162-167.

21. Sebastian, M.P., Khan, M.E., Kumari, K., Idnani, R. (2012) Increasing postpartum contraception in rural India: Evaluation of a community-based behavior change communication intervention. International Perspective on Sexual and Reproductive Health 2(2).

22. Ogburn, J.A., Espey, E., Stonehocker, J. (2005) Barriers to intrauterine device insertion in postpartum women. Contraception 72(6), 426429.

23. Lopez, L.M., Steiner, M., Grimes, D.A., Hilgenberg, D., Schulz, K.F. (2013) Strategies for communicating contraceptive effectiveness. The Cochrane Database of Systematic Review, Issue 4.

24. Arrowsmith, M.E., Aicken, C.R.H., Saxena, S., Majeed, A. (2012) Strategies for improving the acceptability and acceptance of the copper intrauterine device. The Cochrane Database of Systematic Review, Issue 3.

25. Donnelly, K.Z., Foster, T.C., Thompson, R. (2014) What matters most? The content and concordance of patients' and providers' information priorities for contraceptive decision making. Contraception 90(3), 280-287.

26. Critical Appraisal Skills Program. CASP: Making sense of evidence. Available from http://www.casp-uk.net/ [Accessed 23 February 2014]

27. Fraser, R.C., Khunti, K., Baker, R., Lakhani, M. (1997) Effective audit in general practice: a method for systematically developing audit protocols containing evidence-based review criteria. British Journal of General Practice 47(424), 743-746.

28. Sen, G., Grown, C. (2013) Development crises and alternative visions: Third world women's perspectives. Routledge, London.

29. Bolam, A., Manandhar, D.S., Shrestha, P., Ellis, M., Costello, A.M. (1998) The effects of postnatal health education for mothers on infant care and family planning practices in Nepal: a randomised controlled trial. British Medical Journal 316(7134), 805-811.

30. Smith, L.F., Whitfield, M.J. (1995) Women's knowledge of taking oral contraceptive pills correctly and of emergency contraception: effect of providing information leaflets in general practice. British Journal of General Practice 45(397), 409-414.

31. Saeed, G.A., Fakhar, S., Rahim, F., Tabassum, S. (2008) Change in trend of contraceptive uptake-effect of educational leaflets and counseling. Contraception 77(5), 377-381.

32. Afsar, H.A., Younus, M. (2005) Recommendations to strengthen the role of lady health workers in the national program for family planning and primary health care in Pakistan: the health workers perspective. Journal of Ayub Medical Collage Abbottabad 17, 48-53.

33. Hossain, N., Hashmi, H.A. (1998) Family planning myths and facts. Med Channel 4, 21 26.

34. Sikanadar, Q.M., Mehbub, S., Masood, K., Abbasi, M., Butt, N. (2001) Role of male component in family planning. Pakistan Journal of Medical Research 40, 29-31.

35. Thaver, I.H., Lalji, N. (1999) Seeking help for abortion: problems in accessibility and quality in family planning services. Journal of College of Physicians and Surgeons Pakistan 9(1), 8-10.

36. Metson, D., Kassianos, G., Moriarty, J., Norman, D. (1992) Effect of information leaflets on long term recall - useful or useless? British Journal of Family Planning 17, 21-23. 